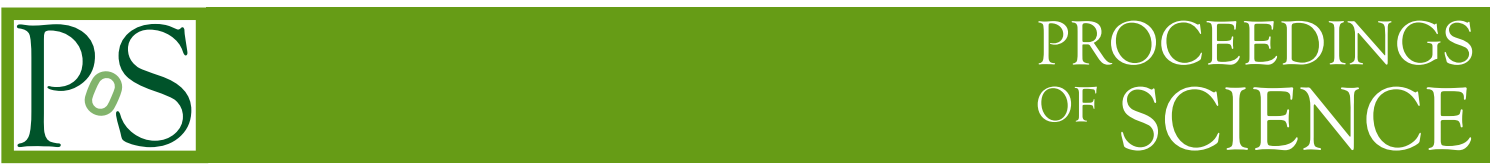

\title{
Extremely fine grained electro-magnetic calorimeter
}

\section{Gerardus Nooren* \\ Institute for Subatomic Physics Utrecht University P.O.B. 80000, 3508 TA Utrecht, the Netherlands}

E-mail: neorendaikhef.n]

\begin{abstract}
Calorimetry at small forward angles at the LHC poses several challenges. The particle density is very high, especially in $\mathrm{Pb}-\mathrm{Pb}$. Further, in the electromagnetic case the discrimination of photons and neutral pions requires extremely fine granularity due to the small angle between the decay photons of the pion. We present a design of a silicon - tungsten calorimeter with Monolithic Active Pixels as sensing element. With a Moliere radius of pure tungsten of $9 \mathrm{~mm}$ it seems overkill to use pixels of $30 . .100 \mu \mathrm{m}$ size. However, in the preshower phase this spatial resolution is helpful. The small pixelsize allows to count pixels to obtain the energy signal without the problem of saturation. Due to the on chip digitization a longitudinal read-out every radiation length becomes feasible. Simulations have shown that a spatial resolution of better than $1 \mathrm{~mm}$ can be achieved, together with a moderate energy resolution. A full depth (20 radiation length) prototype will be put in the testbeam later this year.
\end{abstract}

10th International Conference on Large Scale Applications and Radiation Hardness of Semiconductor Detectors

6-8 July 2011

Florence, Italy

\footnotetext{
* Speaker.
} 


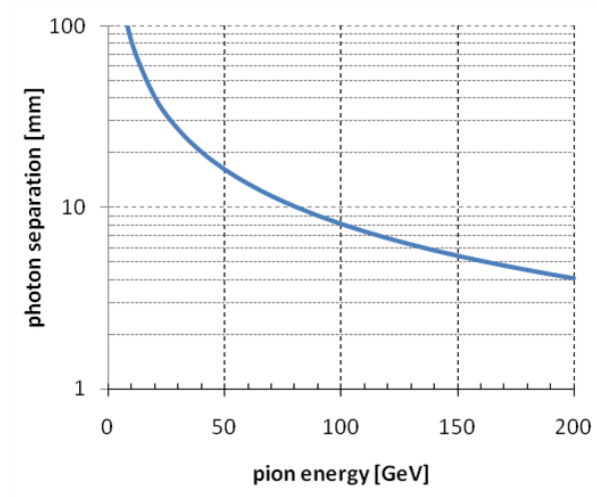

Figure 1: Minimum separation of the two photons from the decay of a neutral pion. The distance to the vertex is $3 \mathrm{~m}$.

\section{Introduction}

Fine grained calorimetry was realised in the past, e.g. in the PAMELA space mission [W], but is not a common technique. The advantages are obvious in tracking and particle identification. Longitudinally, high granularity allows to determine the shower shape which helps to discriminate showering and non-showering particles. Transversely it is a conditio sine qua non in order to discriminate the photons originating from $\pi^{0}$ decay from direct photons. This is especially true at forward angles where energies are high and the distance between the photons becomes very small. Figure. $\square$ shows the smallest distance between the two decay photons at a typical distance of $3 \mathrm{~m}$ from the decay point of the $\pi^{0}$. Clearly, sub-centimetre resolution is needed, at least in the pre- and early shower parts of the calorimeter.

Such a calorimeter would have a lateral resolution of $1 \mathrm{~mm}$ and a longitudinal segmentation of $1 X_{0}$. This leads to an enormous number of channels, which is only feasible with digital calorimetry. In order for digital calorimetry to work the occupancy per pixel should be less than one. With a particle density of $10^{3} \mathrm{~mm}^{-2}$ in the shower core, one requires very small pixels, in the order of 50 .. $100 \mu \mathrm{m}$. A simulation with $0.1 \mathrm{~mm}$ pixels in 24 layers of sensor+absorber is shown in fig. $\mathbb{Z}$

To fully exploit the geometrical resolution obtainable with this small pixelsize, the Moliere radius of the calorimeter should be as small as possible. This leads to the choice of tungsten as absorber and silicon sensors, whilst minimizing the thickness of the electrical connections.

\section{A possible implementation based on MAPS}

Connecting mm-size pixels to front-end electronics in a classical way (e.g. by bump-bonding) is very costly and has never been shown for large surface sensors. The availability of Monolithic Active Pixel Sensors [ [ $[$ ] solves the connection problem between sensor and front-end in an elegant way. Having sensor and front-end electronics on the same chip also reduces the thickness of the construction which leads to smaller effective Moliere radius. A calorimeter based on MAPS was proposed in the context of the CALICE project for ILC [B]], [团]. The last decade intense development

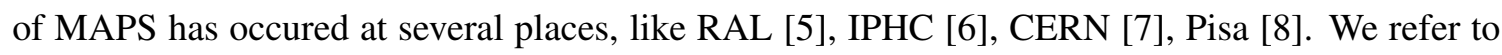
the literature for a full discussion of the various options. In general, points that require special consideration are:

- the high power density inside the sensor requires cooling, also to keep the dark current low; 


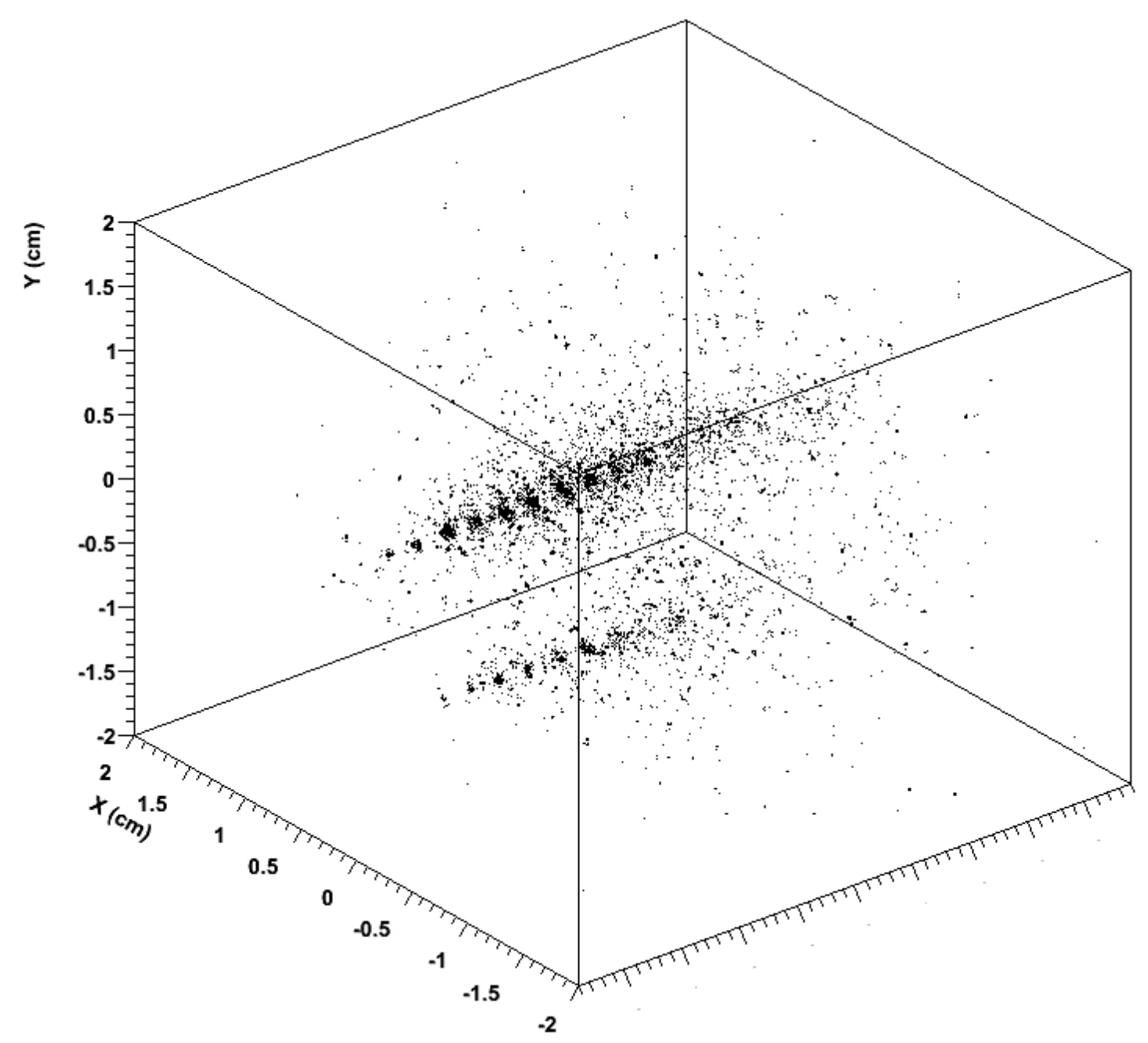

Figure 2: Simulation of two photons from the decay of a neutral pion of $100 \mathrm{GeV}$. The distance to the vertex is $3.5 \mathrm{~m}$. Each point represents a hit in a $0.1 \mathrm{~mm}$ pixel. Z-axis not to scale.

- the charge collection is usually by diffusion which makes the sensor slow and more sensitive to radiation damage.

\subsection{Conceptual design}

The feasibility of such a calorimeter was studied in a conceptual design which aimed to solve two major issues: power density and data volume. Compared to a normal sensor, the active pixel sensor disspates much more heat, typically $90 \mathrm{~mW} \cdot \mathrm{cm}^{-2}$. It was realised that the power can be transported from the chips to the outside by using the rather good heat conductivity of $\mathrm{W}$, namely $170 \mathrm{~W} / \mathrm{m} / \mathrm{K}(\mathrm{Al}: 237 \mathrm{~W} / \mathrm{m} / \mathrm{K})$. As the tungsten is used as absorber anyway, because of its small Moliere radius, it can serve two purposes. The absence of a separate layer of cooling elements leads to a very compact calorimeter, so a small Moliere radius. A typical tower would be composed of layers consisting of tungsten, with the chips glued directly onto it. A thin layer of flexible cables will transport the data to the back of the tower. The perimeter of the $\mathrm{W}$ plate is connected to cooling 
pipes running in the space between towers. This design leads to a heat resistance of $1 \mathrm{~K} / \mathrm{W}$ from chip to cooling water. As the digital areas of chips are dead there is a need for overlap. With the size of MAPS sensors restricted to the typical reticule size of $2 \mathrm{~cm}$ the chance of a shower containing a dead zone would be too high.

For assessing the data volume let's assume a calorimeter at forward region with a surface area of $1 \mathrm{~m}^{2}$. With 24 layers the total silicon area becomes $24 \mathrm{~m}^{2}$, comprising $10^{10}$ pixels of $0.1 \mathrm{~mm}$. Each readout then leads to 10 Gbit. With the rolling shutter providing full frames every $20 \mu \mathrm{s}$, a data rate of $0.5 \mathrm{~PB} / \mathrm{s}$ results. Data reduction is clearly needed and has to be done at various levels:

- select and compress locally, in layer or within the sensorchips. The amount of zero suppression depends on occupancy, which is high for chips at core of the shower and very low far away.

- selection at the end of a tower (16 chips per layer). When the trigger rate is lower than the rolling shutter rate, only frames with trigger will be sent.

- outside the calorimeter.

Despite all this, locally, the bandwidth need remains high because of the high particle density within a shower. This is different from tracker application. It requires high speed cabling, fibers and components at all levels. An implementation with GBT components [U]] components was studied and appeared feasible.

\subsection{Performance}

The performance of such a calorimeter was estimated with simulations of isolated particles (photons, neutral pions). In an experiment, particle densities and the presence of other particle types will influence the performance. This is not the topic of the present study. Another caveat: the simulations are based on GEANT, which was never tested on sub-millimetre scale. Actual particle densities may be higher which may lead to saturation. As regards the sensor, in the simulations presented here, a perfect sensor was assumed: no crosstalk between cells, all energy loss of particle converted to signal.

\subsubsection{Shower separation}

In order to study the separation of nearby showers, photons were simulated in random orientation, but with a fixed distance of $10 \mathrm{~mm}$ from each other. For each of the 100 simulated showers, the hits of corresponding pixels of all layers were summed (fig. [3, left). Then a line was fitted through the data and a histogram was made of the hits projected on this line (fig. [1], centre). Using a simpe approximation with two Gaussians, the peak positions were obtained. These are shown in fig. [1, right. So it appears relatively easy to find the peak position to submillimetre, what about energy?

\subsubsection{Energy measurement}

Knowing the position of the shower axis, as explained above, one can cut out a cylinder along the axis and count the hit pixels. The number of hits is a good measure of the energy, see fig.t. 

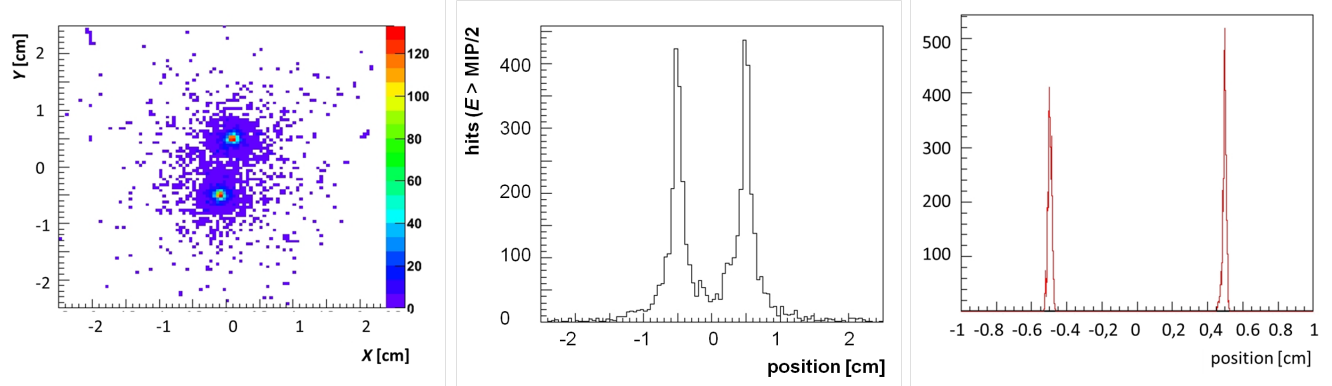

Figure 3: Reconstructed position of two photon showers $10 \mathrm{~mm}$ apart (100 simulations with random orientation). left: Distribution of the hits in all layers in the X-Y plane. Centre: Projection on a single axis. Right: Histogram of the fitted positions.
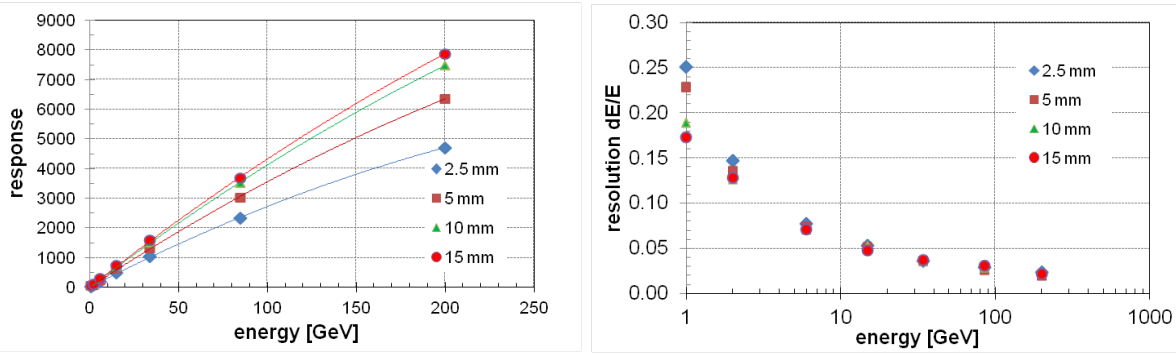

Figure 4: Energy determination by counting pixelhits in a cylindrical volume. The number of hits (left) and the energy resolution (right) as a function of the photon energy for various cylinder radii.

The resolution appears rather insensitive to the diameter of the cylinder, however the linearity does suffer when the diameter becomes smaller.

\section{Prototype}

Due to uncertainties in the simulation but given the great prospects offered by this technique it was decided to build a full prototype to be tested with beam. Small scale prototypes were tested with beam by the RAL group [3] ]. Useful as they are for sensor development, they were too small to test the performance of a calorimeter. For that, one needs a sensor size compatible with the Moliere radius of the test calorimeter. One also needs enough layers to study longitudinal development of a single shower. It was decided to build a prototype based on available MAPS sensors. The choice is limited: most are too small, like TPAC and many MIMOSA. Apart from TPAC, they were designed with tracker application in mind: small pixels, on- board data reduction. We chose PHASE1/2 from IPHC ([Q⿴囗十),[四]). This is the only full size MAPS which allows the continous read-out of all pixels. The small pixel pitch of $30 \mu \mathrm{m}$ allows very fine sampling of the shower core.

\subsection{Objectives}

This protype will act as a technology demonstrator showing the feasibilty of digital calorimetry. It will provide data from which the resolution and Moliere radius can be determined, but also the optimum pixel size. It will show that adequate cooling can be achieved whith a compact detec- 


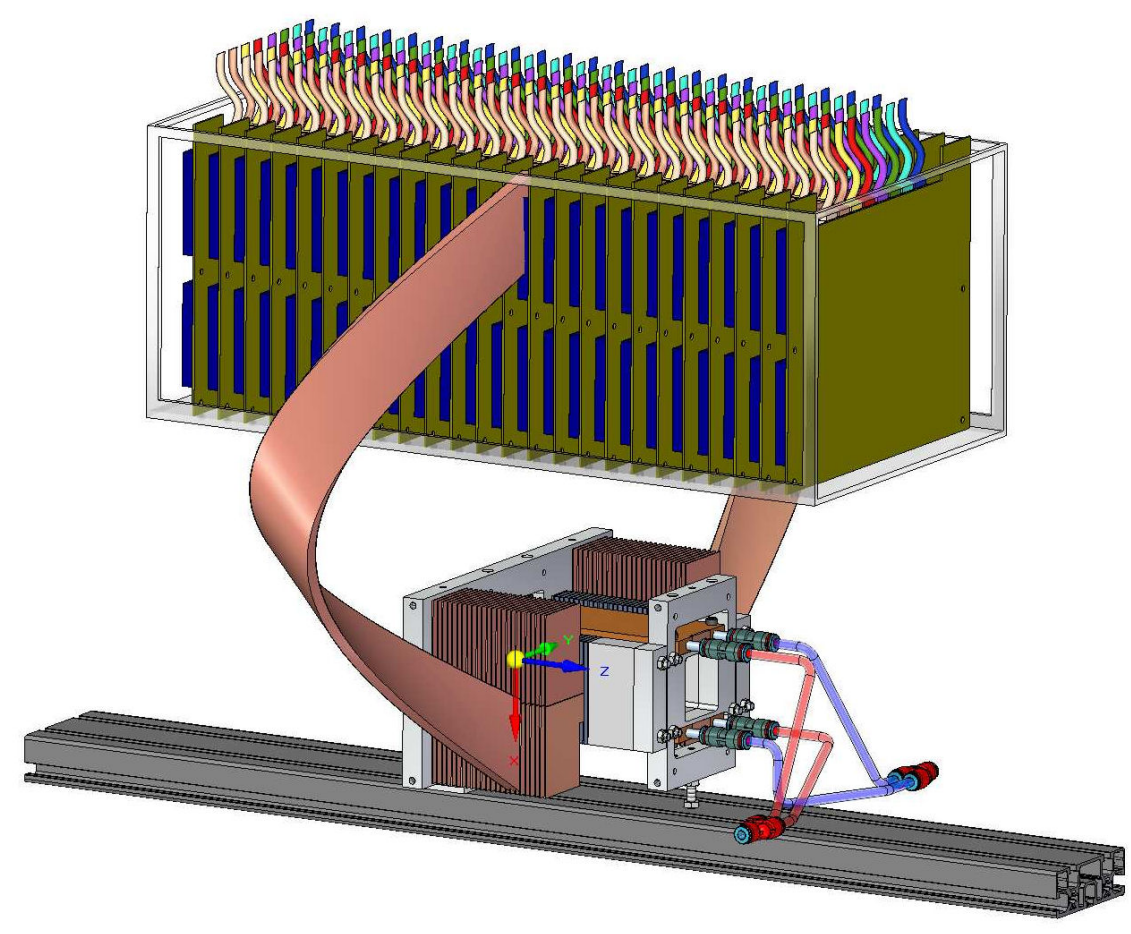

Figure 5: Artist view of the prototype tower. The actual tower is inside, hidden behind cooling and support structures. Only two out of the 96 sensor cables leaving the tower are shown. The box on top is a patch panel containing electronics (buffers and filters). Total length is $0.5 \mathrm{~m}$.

tor while keeping the Moliere radius small. The read-out system will manage the GB/s datarate. The dataset will be used for the study of data volume/flow and data reduction schemes. The prototype will not attempt to validate the sensor, but will provide useful knowledge for the development of a dedicated sensor for calorimetry. Another important objective is the alignment of the large number of sensor chips to the $0.01 \mathrm{~mm}$ level.

\subsection{Features}

The prototype will have 24 layers of 3 or $4 \mathrm{~mm}$ tungsten with 4 PHASE2 sensors in each layer. The dead zones of the sensors in one layer overlap in one direction only. There remains a deadzone of $0.1 \mathrm{~mm}$ between each pair of chips in the other direction. The active area of a layer will be $4 * 4 \mathrm{~cm}^{2}$. The total thickness of a sensor layer, including the overlap, is $1 \mathrm{~mm}$, thanks to the thinning down to $120 \mu \mathrm{m}$ of the sensor chips. This leads to a Moliere radius of better than $14 \mathrm{~mm}$, which makes the tower wide enough to study the lateral shower development. Already this small prototype needs to be watercooled, the dissipation will be $70 \mathrm{~W}$ in $150 \mathrm{~cm}^{3}$.

The PHASE2 sensors feature $640 * 640$ pixels with $30 \mu \mathrm{m}$ pitch in a high resistivity $(400 \Omega \mathrm{cm}$ ) epilayer of 15 and $20 \mu \mathrm{m}$ thickness. The $1 \mathrm{MHz}$ rolling shutter implies an integration time of $640 \mu \mathrm{s}$. Multiple outputs at $160 \mathrm{MHz}$ clock allow full read out of all frames. As each event contains $39 \mathrm{M}$ pixels, the raw data rate of the prototype will be $61 \mathrm{~Gb} / \mathrm{s}$. Several FPGAs will be used to manage this, see fig. 6 . As the average occupancy is expected to be at the precentage level, 


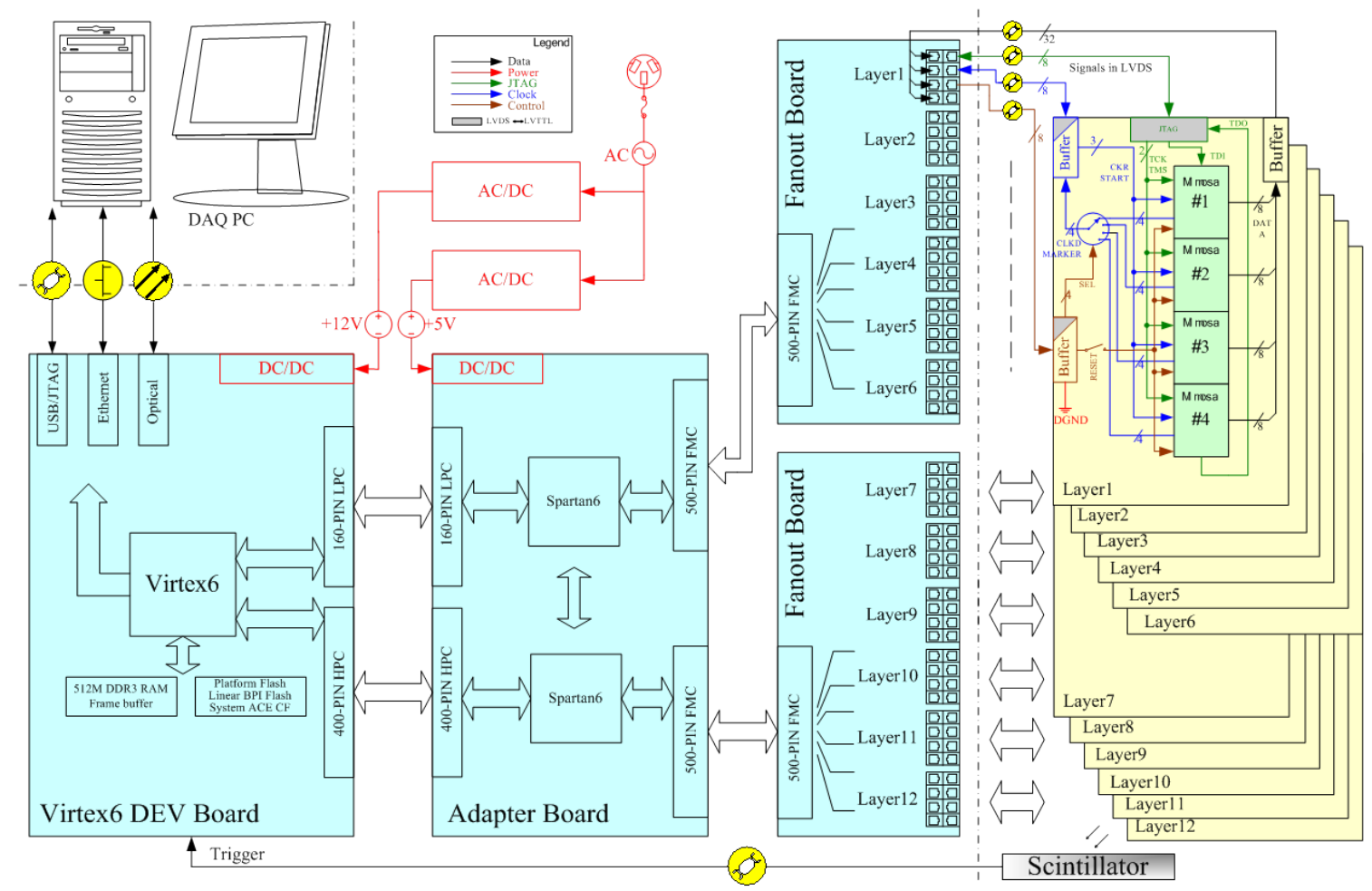

Figure 6: Schematic of the read-out electronics for the prototype tower. Only the circuits for 12 layers are shown.

zero suppression is planned at the pixel and line level and frames without trigger will be discarded. A big local buffer memory will allow to profit from the small duty factor of PS/SPS. Because each sensor has its own cable, the set-up is dominated by the interconnections (fig. [1]).

\section{Conclusion}

An EM calorimeter with fine sampling and pixel counting is within reach and would open new possibilities for particle identification in calorimetry. Because of the uncertainties in simulations: shower development on very small scale, the importance of low-energy particles and unknowns in the charge collection, Utrecht University/Nikhef and Bergen University are constructing a prototype calorimeter. It features extremely fine segmentation both longitudinal and tranversal and allows full data read-out. Beamtest at CERN PS is planned in November 2011, at SPS in 2012. This detector is an option for a possible future forward calorimeter in ALICE.

\section{References}

[1] Boezio et al., A high granularity imaging calorimeter for cosmic-ray physics, Nucl. Inst. Meth. A487 (2002), 407.

[2] R. Turchetta et al., A monolithic active pixel sensor for charged particle tracking and imaging using standards VLSI CMOS technology, Nucl. Inst. Meth. A458 (2001), 677. 
[3] P.F. Dauncey, Performance of CMOS sensors for a digital electromagnetic calorimeter, PoS (ICHEP 2010) 502

[4] J.A. Ballin et al., A MAPS-based Digital electromagnetic Calorimeter for the ILC, Proceedings of 2007 International Linear Collider Workshop, Hamburg, Germany (2007), arXiv:0709.1346

[5] P.F. Dauncey et al., Design and performance of a CMOS study sensor for a binary readout electromagnetic calorimeter, JINST 6 (2011) P05009

[6] G. Voutsinas et al., Nuclear Physics B (Proc.Suppl.) 215 (2011) 48

[7] J.D. Segal et al., Second generation monolithic full-depletion radiation sensor with integrated CMOS circuitry, SLAC-PUB-14318 (2010)

[8] S. Bettarini, this conference

[9] A. Brogna et al., Manual PHASE1, IPHC Strasbourg

[10] M. Szelezniak, CMOS pixel vertex detector for STAR, PoS (VERTEX 2008) 032

[11] https://espace.cern.ch/GBT-Project/default.aspx 